\title{
4
}

\section{Content-based Video Retrieval Based on Similarity of Camera Motion}

\author{
Hitoshi Endoh and Ryoji Kataoka \\ Information-base Project, NTT Cyber Space Laboratories \\ 1-1 Hikarinooka Yokosuka-Shi Kanagawa 239-0847, Japan \\ E-mail: \{endoh, kataoka\}@dq. isl.ntt.co.jp
}

\begin{abstract}
This paper proposes a method for content-based sports video retrieval using camera work information. Since particular camera work for a typical scene exists in sports videos, camera work transition becomes an effective cue for retrieving a sports scene based on its content. The proposed method extracts a series of camera parameters from both a user-specified scene of a retrieval key and a video stream of a retrieval target, and detects scenes having content similar to that of the key from the target by applying continuous DP matching. The method was evaluated using a video stream of a baseball game. RecallPrecision curves make its effectiveness clear.
\end{abstract}

Key words: Video, MPEG, DP matching, Content-based retrieval

\section{INTRODUCTION}

Advanced video compression technology such as the MPEG videoencoding scheme makes it possible to store large digital video archives in computer systems. As the quantity of the video archives increases, it becomes impractical for video users to search for their favorite scenes from the archives by watching them one by one. A video database system is expected to solve this problem and a great deal of research has recently been done on content-based video retrieval techniques. To realize content-based video retrieval, semantic information representing a scene content should be assigned to each scene as an index. It is, however, impractical to assign all the semantic information by handwork since each video stream generally includes an enormous amount of scenes and semantics. Therefore, video retrieval based on the similarity of video features is a reasonable approach 
since such features as a color histogram, camera work information and object motions can automatically be extracted from video streams (Miyamori et al., 1998)(VisualSEEk)(Chang et al., 1997)(Smith et al., 1998)(Mohan, 1998).

This paper proposes a method of content-based sports scene retrieval using camera work information as a video feature. In broadcasting sports programs, semantically the same scenes tend to be captured by the same camera work. For example, home run scenes in baseball programs have a typical transition of camera work as follows: follow the ball struck by the batter, zoom in on the stands where the ball falls in and follow the batter running around the diamond. In soccer programs, the following typical camera work exists in corner kick scenes: follow the ball kicked from the corner to the front of the goal post and zoom in on the front of the goal post. Therefore, camera work transition is expected to be an effective cue for retrieving similar highlight scenes from sports video programs.

The proposed method allows users to input a sample scene having some semantics as a retrieval key. For example, when a user wants to retrieve home run scenes from a baseball game program, he or she inputs a typical sample of a home run scene. Camera work information is then extracted using motion vectors in an MPEG encoded video stream from both the userspecified sample and the target program. Extracted information consists of a series of camera parameters. Similar scenes are detected using continuous DP matching between the obtained series of camera parameters.

The rest of the paper is organized as follows: Section 2 discusses related research work that has been reported. Section 3 describes the proposed method for content-based sports scene retrieval using camera work information. Section 4 presents experimental results obtained in applying scene retrieval to a baseball game program. Finally, concluding remarks appear in Section 5.

\section{RELATED RESEARCH WORK}

Several content-based scene retrieval methods using video features have been proposed so far. One of the methods defines a relationship rule between semantics of a scene and its structure of events (Miyamori et al., 1998). In this method, each event of a soccer game is defined using a short-time action description. The description is composed of a player's ID, position, and actions like (WHO, WHERE, WHAT). Relationship rules can be described as "heading shoot $=$ (field player, in front of goal, jump $)+($ keeper, in front of goal, dive)" and "corner kick = (field player, corner, kick)". When a user inputs a semantic keyword about a scene he or she is interested in, it is 
interpreted into a set of events based on a rule dictionary. Since each description is also related to a scene's physical position on a video stream, scenes having user-specified semantics can be obtained from the interpreted events. To automatically relate short-time action descriptions with their physical scene positions, advanced image processing and recognition techniques are required to extract and analyze detailed features of a video stream. Considering the actual current level of these techniques, this method is impractical since it forces users to define almost all the relationships by handwork.

Another existing method allows users to directly input a retrieval key with features. It extracts features such as the color, shape, and moving direction of objects in a video stream and constructs an index from them. Users request scenes they are interested in by specifying object information like "a red object moving to the left". This method is a reasonable approach since it is based on video features that can automatically be extracted using current image processing techniques. It, however, has a drawback in that it is difficult to represent complicated movement such as that which occurs in home run scene in a baseball game as a retrieval key.

Another method has been proposed to eliminate the above-mentioned drawback, in which a sample scene having complicated features itself can be used as a retrieval key (Smith et al., 1998). It extracts color information from both a user-specified scene and video streams in a database, and constructs feature histograms from each bit of color information. It then detects the scenes similar to the user-specified one by matching those histograms. Since feature histograms do not include information about the appearance order of features in a video stream, however, this method cannot detect scenes based on the similarity of motion transition. Furthermore, it cannot consider differences in scene length between a user-specified one and similar ones in a video database. As a result, for example, a slow motion replay of a userspecified home run scene cannot be detected as a similar scene.

This paper proposes an effective method for content-based sports scene retrieval by solving the above-mentioned problems in the existing methods. It takes both motion transition similarity and differences in scene length into consideration in detecting scenes similar to a user-specified one. 


\subsection{Outline of the Process}

Figure 1 shows the outline of the proposed method. The individual steps can be summarized as follows:

1. Specification of a Retrieval Key

A user specifies a sample scene he or she wants to retrieve as a retrieval key. For example, when a user wants to retrieve home run scenes from a baseball game program, he or she inputs a typical sample of a home run scene.

2. Feature Extraction

Camera parameters are extracted based on the optical flow from both the user-specified scene and a target video. Camera parameters give global and local motion. Global motion represents the movement of a background caused by the movement of a camera. Local motion represents the movement of objects captured in a video. In this paper, we use motion vectors including an MPEG encoded video stream as optical flow to obtain camera parameters.

\section{Similar Scene Detection}

Scenes similar to the retrieval key are detected by feature matching between the extracted two series of camera parameters. Though several methods have been proposed to perform matching between two series of features, we adopt the continuous DP matching method since it effectively detects similar scenes by taking both motion transition similarity and differences in scene length into consideration. The continuous DP matching detects many overlapped scenes. Therefore, our method removes closely overlapped scenes from the matching results and gives only meaningful scenes.

\subsection{Feature Extraction}

To estimate camera parameters (i.e. information on the degree of pan and zoom operations) based on optical flow of video images, we adopt the technique mentioned in (Meng et al., 1996) that uses motion vectors in an MPEG encoded video stream as optical flow. Given the motion vectors (u, v) for each macroblock in a P-picture, the following equation is generally satisfied if the vector represents the movement of a background (i.e. global motion) through the operations of a fixed position camera: 


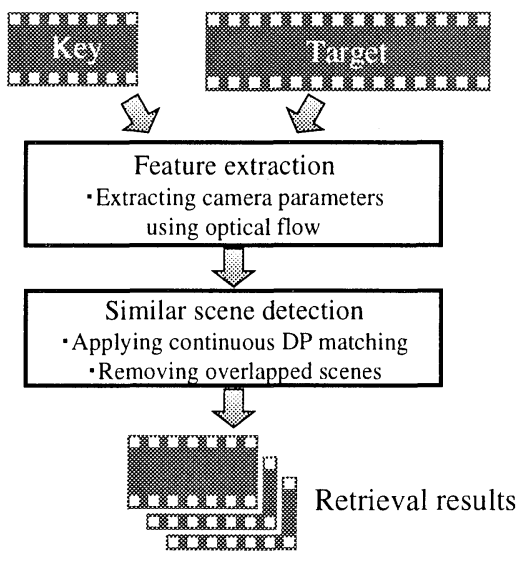

Figure 1. Outline of proposed method.

$$
\left(\begin{array}{l}
u \\
v
\end{array}\right)=G_{z}\left(\begin{array}{l}
x \\
y
\end{array}\right)+\left(\begin{array}{l}
G_{x} \\
G_{y}
\end{array}\right)
$$

where $(x, y)$ are the coordinates of the macroblock, $G_{x}$ is the degree of a horizontal pan operation, $G_{y}$ is the degree of a vertical pan operation, and $G_{z}$ is the degree of a zoom operation. For each P-picture, $\left(G_{x}, G_{y}, G_{z}\right)$ is estimated using the Least Square estimation to minimize the error between the motion vectors estimated in (1) and the actual motion vectors obtained from an MPEG video stream. Not all motion vectors in an MPEG video stream, however, represent global motion. Consequently, if the difference between estimated and actual motion vectors is greater than a certain threshold, the actual one is excluded from the Least Square estimation. The estimation is repeated until no exclusive vector is found.

Motion vectors excluded in the above estimation are considered as the vectors representing the movement of objects (i.e. local motion). The average value of these vectors $\left(\mathrm{L}_{\mathrm{x}}, \mathrm{L}_{\mathrm{y}}\right)$ are also treated as video features and used for feature matching. Therefore, the features extracted from the i-th Ppicture in a MPEG video stream are represented as follows:

$$
f(i)=\left(G_{x}, G_{y}, G_{z}, L_{x}, L_{y}\right)
$$

The series of features $F_{S}$ and $F_{T}$ obtained from a retrieval key $S$ and a target video stream $\mathrm{T}$ are represented respectively as follows:

$$
F_{S}=f_{S}(1) \prec f_{S}(2) \prec \cdots \prec f_{S}\left(N_{S}\right)
$$




$$
F_{T}=f_{T}(1) \prec f_{T}(2) \prec \cdots \prec f_{T}\left(N_{T}\right)
$$

where $\mathrm{N}_{\mathrm{S}}$ and $\mathrm{N}_{\mathrm{T}}$ are the number of P-pictures in $\mathrm{S}$ and $\mathrm{T}$, respectively.

\subsection{Feature Matching}

Our content-based sports scene retrieval is based on the generality that semantically the same scenes tend to be captured by the same camera work in a broadcasting sports program. Therefore, the appearance order of camera parameters is very important to detect similar sports scenes. Furthermore, a slow motion replay scene should always be detected as a similar one when its original one is given as a retrieval key since these semantics are obviously the same. Therefore, a feature matching method must be able to consider the differences in length between original and replay scenes. Considering these requirements, we adopt continuous DP matching as a feature matching method.

Continuous DP matching compares $\mathrm{F}_{\mathrm{S}}$ with a partial section of $\mathrm{F}_{\mathrm{T}}$ while expanding or contracting $F_{S}$, and determines the correspondence of $f_{S}(i)$ and $\mathrm{f}_{\mathrm{T}}(\mathrm{j})$ as the pattern distance $\mathrm{D}(\mathrm{k})$ representing the similarity of features is minimized. When $f_{S}\left(N_{S}\right)$, i.e. the end of $F_{S}$, is corresponded to a $f_{T}(k)(1 \leqq k$ $\left.\leqq N_{T}\right), D(k)$ is obtained as the following recursive formula:

$$
\begin{aligned}
& D(k)=\frac{1}{k-k^{\prime}+N_{S}} g\left(k, N_{S}\right) \\
& g(\tau, 1)=\infty\left(2 \leq \tau \leq N_{S}\right) \\
& g(1, \tau)=2 d(1, \tau)\left(1 \leq \tau \leq N_{T}\right) \\
& g(i, j)=\min \left(\begin{array}{l}
g(i-1, j-1)+2 d(i, j) \\
g(i-1, j-2)+3 d(i, j) \\
g(i-2, j-1)+3 d(i, j)
\end{array}\right)
\end{aligned}
$$

where $k^{\prime}$ is the value of $j$ when $i$ is 1 , i.e. $f_{S}(1)$ is corresponded to $f_{T}\left(k^{\prime}\right)$. $d(i, j)$ is the distance between $f_{S}(i)$ and $f_{T}(j)$. In this paper, it is defined as follows: 


$$
\begin{aligned}
& d_{\text {pan }}=\left(G_{x}(i)-G_{x}(j)\right)^{2}+\left(G_{y}(i)-G_{y}(j)\right)^{2} \\
& d_{\text {zoom }}=\left(G_{z}(i)-G_{z}(j)\right)^{2} \\
& d_{\text {local }}=\left(L_{x}(i)-L_{x}(j)\right)^{2}+\left(L_{y}(i)-L_{y}(j)\right)^{2} \\
& d(i, j)=\sqrt{\omega_{1} d_{\text {pan }}+\omega_{2} d_{\text {zoom }}+\omega_{3} d_{\text {local }}}
\end{aligned}
$$

The degree of influence on global and local motions can be adjusted by the values of $\omega_{1}, \omega_{2}$ and $\omega_{3}$. Since pan and zoom operations occur simultaneously, the degree of influence on $d_{p a n}$ and $d_{z o o m}$ can be varied independently by the value of $\omega_{1}$ and $\omega_{2}$.

$\mathrm{D}(\mathrm{k})$ represents the similarity between a user-specified scene $\mathrm{S}$ and a partial section of $\mathrm{T}$ bounded by k' and $\mathrm{k}$. The higher the similarity is, the smaller the value of $D(k)$ becomes. Since obtained partial sections corresponding to all $\mathrm{D}(\mathrm{k})$ 's for every $\mathrm{k}\left(1 \leqq \mathrm{k} \leqq \mathrm{N}_{\mathrm{T}}\right)$ considerably overlap each other in general, redundant sections having large $\mathrm{D}(\mathrm{k})$ are removed from the matching results.

\section{EXPERIMENTS AND EVALUATIONS}

\subsection{Prototype Overview}

We implemented the proposed method on a prototype system to determine the method's validity. Figure 2 shows the system overview. The main components are the pre-processing module, the GUI module, the similar scene detection module, the sample scene selection module and the parameter setting module.

The GUI module consists of the query composer and the result viewer. It allows a user to select a target video stream, specify a scene using a retrieval key and set the parameters of the feature matching. It is implemented using CGI and runs on a Web browser. 


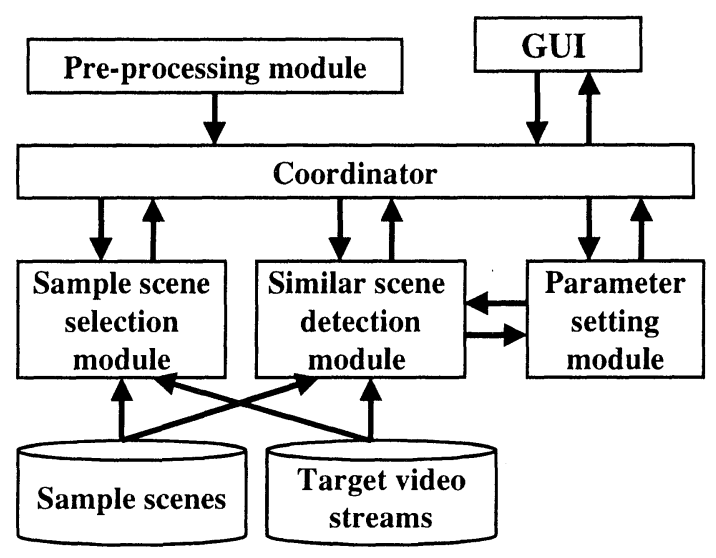

Figure 2. Prototype system overview.

The parameter setting module sets the parameters of $\omega_{1}, \omega_{2}, \omega_{3}$ mentioned above and the extracting interval of P-pictures explained below. These are referred to in the similar scene detection module.

The sample scene selection module allows a user to specify a scene using a retrieval key. A user can select a key from a sample scene database or make it from a target video stream by using the scene cutoff tool in this module. A user can also use the retrieved results as retrieval keys.

The similar scene detection module consists of the feature matching and the redundancy eliminating components. The feature matching component performs the continuous DP matching between two series of features. The redundancy eliminating component removes closely overlapped sections and sections having large $\mathrm{D}(\mathrm{k})$ from the matching results.

The pre-processing module extracts features from a target video stream in advance since it takes a long time to extract features from a large video stream. For example, it takes about 20 minutes for a video stream of an hour in length under the configuration described below. On the other hand, feature extraction for a retrieval key is performed on demand since a retrieval key is generally short and the extraction takes very little time.

Figure 3 shows a screen shot of the prototype system. The system configuration is as follows:

- PC: PentiumII 400MHz, 256MB memory

- OS: WindowsNT4.0

- Browser: Internet Explorer

At the upper left area in Figure 3, a user selects a scene using a retrieval key and previews it. At the upper right area, the user selects a target video stream. Using the buttons in the upper center area, the user sets the 
parameters of the feature matching and starts scene retrieval. Retrieval results are shown in the lower area.

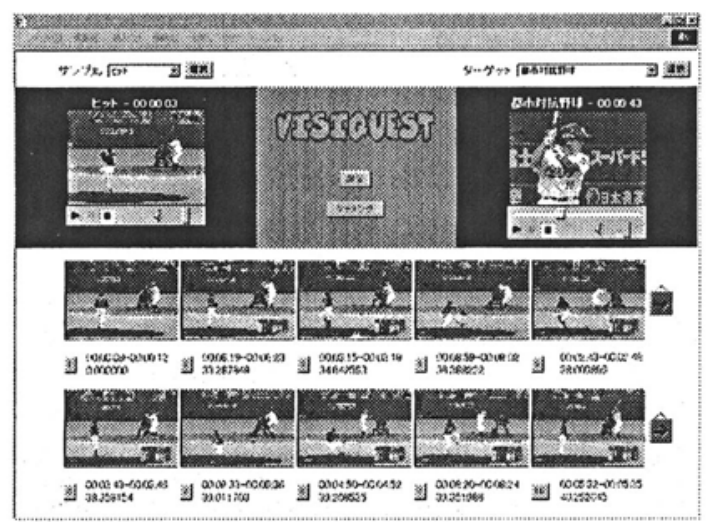

Figure 3. Screen shot of the prototype system

\subsection{Application to a Baseball Game Program}

The efficiency of the proposed method was verified by applying it to a broadcast baseball game program. The program used a target video stream of about 1 hour and 40 minutes in length or about 1 GB in the MPEG-1 compression having 171,152 frames. It includes 46,746 P-pictures (i.e. $\mathrm{N}_{\mathrm{T}}=$ 46,746). Two samples of home run and infield grounder scenes were selected as retrieval keys from the target. Table 1 shows the details of the retrieval keys. Slow-motion replays of original scenes are also counted as the number of the identical semantic scenes.

Table 1. Details of the retrieval keys

\begin{tabular}{lcc}
\hline & Home run & Infield grounder \\
\hline Picture size & $352 \times 240$ & $352 \times 240$ \\
Scene length & $18 \mathrm{sec}$ & $7 \mathrm{sec}$ \\
Number of P-pictures & 148 & 63 \\
Number of identical semantic & 13 & 11 \\
scenes & & \\
\hline
\end{tabular}

Experiments were performed for the following three cases: (a) $\omega 1: \omega 2$ : $\omega 3=1: 1: 1$, (b) $\omega 1: \omega 2: \omega 3=1: 1: 0$, and (c) $\omega 1: \omega 2: \omega 3=0: 0: 1$. In (a), global and local motions exert equivalent influence in the calculating of the similarity of scenes. On the other hand, (b) considers only global motion and (c) only local motion. 
Figures 4 and 5 show the Recall-Precision curves obtained from the results of scene retrievals using the above-mentioned keys. In the home run scene retrieval, the (a) and (b) give better curves than (c). Furthermore, the curve for (a) is almost the same as that for (b). This means that global motion is the main factor in the retrieval of home run scenes. This shows the validity of our idea that semantically the same scenes tend to be captured by the same camera work in a broadcast sports program.

The precision rate in Figure 4 becomes worse when the recall rate is greater than 0.8 . This was due to the fact that three of the 13 home run scenes in the target program were captured with irregular camera work, e.g. the camera followed the flight of the ball from the outfield stands. This shows that a variety of samples for the same semantic scene should be prepared to enhance the precision and recall rates in our method.

The retrieval result of home run scenes clarified the efficiency of continuous DP matching since the slow-motion replay scenes of the given retrieval key were unfailingly detected as very similar scenes. Many scenes of outfield fly balls were also included; this is because the camera motion for capturing outfield fly balls is similar to that for capturing home runs.

In field grounder scene retrieval, on the other hand, (b) gives a worse Precision-Recall curve than (a). The degree of camera work in an infield grounder scene is smaller than that in a home run scene. Furthermore, an infield grounder scene generally includes typical object motion such as an infielder throwing the ball to first base. Therefore, local motion is also useful for the content-based scene retrieval in the proposed method.

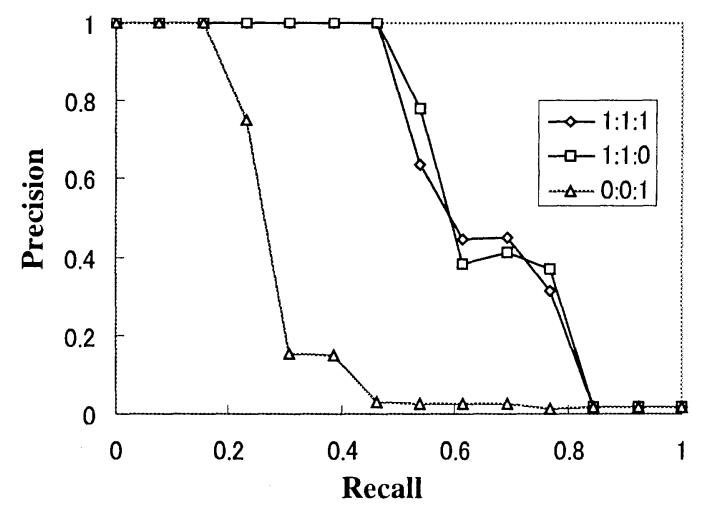

Figure 4. Precision-Recall curve of home run scene retrieval 


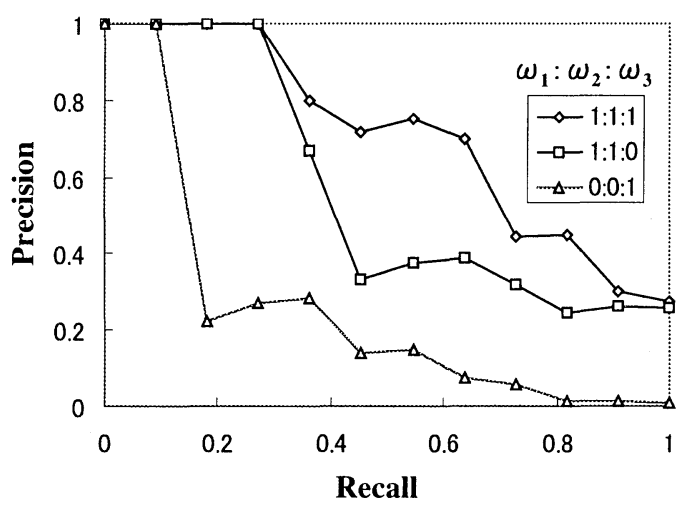

Figure 5. Precision-Recall curve of infield grounder scene retrieval

\subsection{Enhancement of Response Time}

In the previous subsection, all features extracted from P-pictures in both retrieval key and target video stream are applied to the feature matching. Since processing time of the feature matching increases in proportion to the number of features, the number of P-pictures extracted should be as small as possible to enhance the response time of scene retrieval. The smaller the number of extracting P-pictures is, however, the worse the recall and precision rates become. The experiments described in this subsection clarify that how the number of extracted P-pictures affects the recall and precision rates of similar scene retrieval in our method.

The Recall-Precision curves were obtained while the P-picture extraction interval was varied from 1 to 5 . An extracting interval of 5 means, for example, that features are extracted from every fifth P-picture in an MPEG video stream. The same infield grounder scene and the same baseball game program used for the experiments described in the previous subsection were used for a retrieval key and a target video stream, respectively. The results obtained are shown in Figure 6. Almost the same curves were obtained when the extracting intervals were set to $1,2,3$ and 4 . For an interval of 5, however, the curve became worse than for the other cases. This is likely caused by the structure of an MPEG encoded video stream, i.e. one that consists of multiple GOP's (Group Of Pictures). A GOP generally includes four P-pictures as shown in Figure 7. Therefore, at least one P-picture in each GOP is used for feature extraction when the extracting interval is set to between 1 to 4 . In these cases, the lack of features in global and local motion is considered to have little significance since each P-picture is derived from 
the same I-picture in the GOP through the motion compensation processing. On the other hand, it extracts a comparatively strong effect for an interval of 5 since many GOP's are skipped in the feature extraction. Though our method can be applied in the same manner when features of B-pictures are also extracted and used for the feature matching in addition to those of Ppictures, these results show that such additional features would not enhance the recall and precision rates to any significant degree.

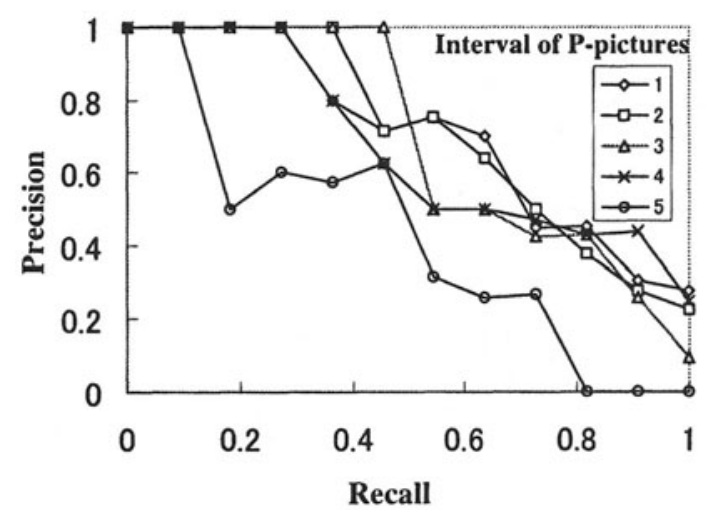

Figure 6. Effectiveness of P-picture extraction interval



Figure 7. GOP structure

Processing time of the feature matching varied as shown in Figure 8. These results are understandable since the processing cost of the feature matching is $\mathrm{O}\left(\mathrm{N}_{\mathrm{S}} * \mathrm{~N}_{\mathrm{T}}\right)$, where $\mathrm{N}_{\mathrm{S}}$ and $\mathrm{N}_{\mathrm{T}}$ are the number of P-pictures extracted from a retrieval key and a target video stream, respectively. The 
response time can be considerably shortened when the P-picture extraction interval is set to between 2 and 4, while maintaining the recall and precision rates at almost the same as those when all P-pictures are used in the feature matching.



Figure 8. Processing time of the feature matching

\subsection{Effectiveness of Local Motion}

Another experiment was performed to clarify the effectiveness of using local motion in the proposed method. For the same baseball program target, a video clip in which a person turns his hand around twice in front of a fixed camera is given as a retrieval key.

For $\omega 1: \omega 2: \omega 3=1: 1: 1$, the detected scenes with high similarity mostly contain images of a batter making two practice swings with his bat. On the other hand, for $\omega 1: \omega 2: \omega 3=1: 1: 0$, scenes containing object motion similar to that in the retrieval key cannot be detected. These results show the proposed method has the ability to retrieve scenes on the basis of object movement similarity as well.

\section{CONCLUSIONS}

This paper proposed a method of content-based sports scene retrieval using camera work information as a video feature. The method extracts a series of camera parameters from both a user-specified scene of a retrieval key and a video stream of a retrieval target using motion vectors in MPEG encoded video streams. Continuous DP matching is adopted for the feature matching between a retrieval key and a target stream since it takes both 
motion transition similarity and differences in scene length into consideration.

The method was evaluated using a broadcast baseball game program. The result showed the validity of our idea that semantically the same scenes tend to be captured by the same camera work in broadcasting sports programs. It was verified that the continuous DP matching could adapt to differences in scene length since slow-motion replays of a given home run scene were unfailingly detected as very similar scenes. It also showed that local motion information was effective in such cases as the retrieval of infield grounder scenes.

To enhance the response time, we studied how the number of P-pictures extracted affected the recall and precision rates in our method. The results obtained showed that almost the same recall and precision rates could be obtained even if only every fourth P-picture in an MPEG video stream were used in the feature matching, while reducing the response time to about 1/16 of the original value.

Even if the response time can be further shortened by decreasing the number of P-pictures extracted, it becomes longer in proportion to the length of a target video stream. Therefore, ideas on how to solve this problem should be studied to apply our method to large video databases. It will also be necessary to study the effectiveness of additional features such as color information and scene composition in future.

In the near future, a home server system that can store large quantities of digital video data will come into common use. It makes it possible that people can watch any TV programs broadcasted in a whole week at any time they want even if they forgot to reserve video recording of their favorites. Video summarizing function becomes very important in such situation since they need to find their favorites from enormous TV programs stored in a home server. The proposed method will be helpful to realize the function since it can automatically generate user's favorite digest by registing a sample set of his or her interested scenes to the server.

\section{REFERENCES}

Hisashi Miyamori et al.(1998) Proposal of Content-based Video Retrieval Method using

Short-time Action Descriptions in a scene, Proc. MIRU'98 (in Japanese).

http://disney.ctr.columbia.edu/VisualSEEk/VisualSEEk_description.html.

S.Chang et al.(1997) VideoQ: An Automated Content Based Video Search System Using

Visual Cues, Proc. ACM Multimedia'97.

Gravin Smith, Hiroshi Murase, Kunio Kashino(1998) QUICK AUDIO RETRIEVAL USING

ACTIVE SEARCH, Proc. ICASSP'98.

Rakesh Mohan(1998) VIDEO SEQUENCE MATCHING, Proc. IEEE Int Conf Acoust

Speech Signal Process'98. 
Nakagawa Sei-ichi(1983) A RECOGNITION METHOD OF CONNECTED SPOKEN WORDS WITH SYNTACTICAL CONSTRAINTS, Proc. IJCAI'83.

J. Meng and S.Chang(1996) CVEPS - A Compressed Video Editing and Parsing System, Proc. ACM Multimedia'96.

\section{BIOGRAPHIES}

Hitoshi Endoh received Bachelor degree in physics from Tokyo Institute of Technology, Japan, in 1995 and Master degree in general systems study from University of Tokyo, Japan, in 1997. He then joined NTT Information and Communication Systems Laboratories where he has been engaged in research on multimedia database systems. He is currently a researcher in NTT Cyber Space Laboratories. Mr. Endoh is a member of Information Processing Society of Japan.

Ryoji Kataoka received the B.E. and M.E. degrees in electronic engineering from Chiba University, Japan, in 1985 and 1987, respectively. He then joined NTT Communications and Information Processing Laboratories where he has been engaged in research on database management systems and multimedia information systems. He is currently a Senior Research Engineer in NTT Cyber Space Laboratories. Mr. Kataoka is a member of Information Processing Society of Japan and the Institute of Electronics, Information and Communication Engineers. 